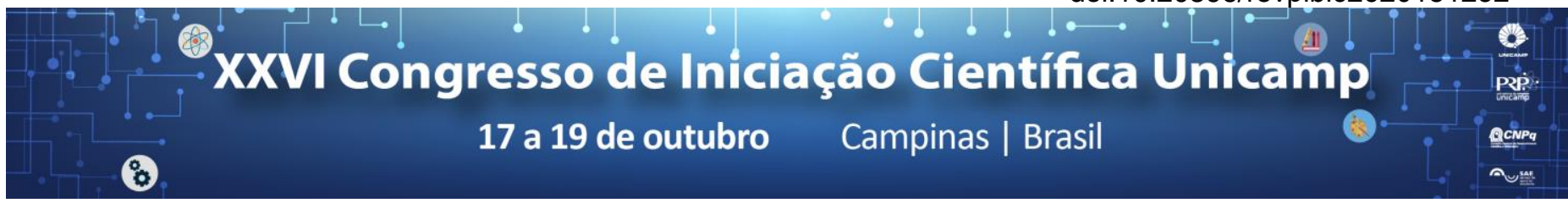

\title{
Development of a cryogenic system for a UHV manipulator for a light detection device in a LT-STM.
}

\section{Ronaldo R. F. Vieira*, Yves M. Auad, Luiz F. Zagonel.}

\begin{abstract}
This project aims to develop a cryogenic system for a UHV (Ultra-High Vacuum) manipulator that is currently under development in our group and that will be installed inside a low temperature scanning tunneling microscope (LT-STM). This advice will be used as a light detector to collect the Luminescence in the STM scan, and since the equipment will operate in low temperatures, it is necessary that this advice also operate in low temperatures so that it won't cause thermal instability which would prefent image acquisition due to thermal drifts.
\end{abstract}

\section{Key words:}

UHV, LT-STM, Cryogenic, instrumentation.

\section{Introduction}

Luminescence in STM can occur due to tunneling current between sample and tip during the sample imaging. However, to collect such light, it is necessary input an advice inside the STM close to the tunnel junction.

The problem arises because, in our case, the light collector will stay $300 \mu \mathrm{m}$ of distance from the sample, and the sample temperature is about $13 \mathrm{~K}$ (if cooled by liquid Helium) while the manipulator is about $297 \mathrm{~K}$. This proximity, by radiation, can provoke a thermal instability in the sample and bad data collections due to thermal drift.

Thus, this project aims to develop a cryogenic system for a UHV manipulator that reach temperatures about $77 \mathrm{~K}$ (Liquid Nitrogen flux) until $20 \mathrm{~K}$ (Liquid Helium flux), and integrate this advice in a low temperature scanning tunneling microscope (LT-STM).

\section{Results and Discussion}

Through the CFD Autodesk, we have made some thermal simulations of sample and tip model, in order to show the system temperature instability. As shown in image 1. It shows that the light collector will warm the sample significantly.

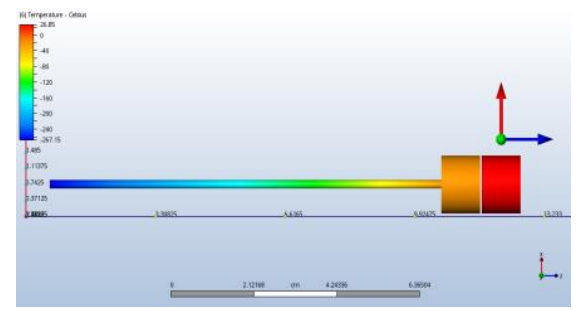

Image 1: Thermal simulation of sample and tip model.

Therefore, to create a first prototype, were studied the materials properties in UHV environment. Were realized a delicate choice of materials, because to obtain the ultrahigh vacuum depends strictly of that choice.

After realize the materials choice, were created a series of solutions thinking in all possibilities, as machining techniques, viability, efficiency, etc. Subsequently all considerations, we have found a model that attended all requirements and created a first prototype. As shown in image 2.

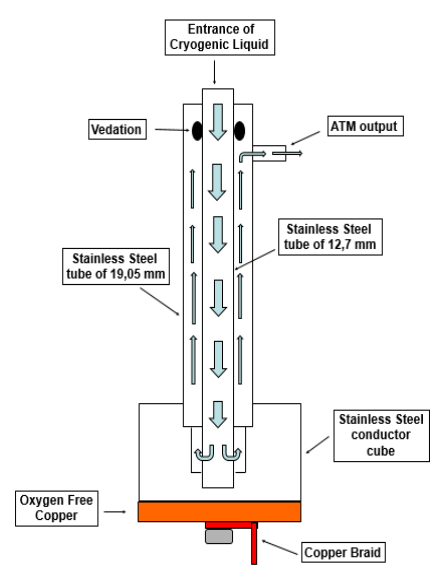

Image 2: First prototype.

This prototype were selected due to its robustness, which will provide less vibration to the system; its facility to machining and assembly; and through the flux of cryogenic liquid will be possible to obtain a better efficiency in the heat exchange.

Besides that, the temperature will be monitored with resistive temperature sensor PT100, (because it is compatible with UHV environment and efficient in wide temperature range) in three crucial points, manipulator, copper block and piezoelectric.

\section{Conclusions}

The manipulator UHV cooling system opened a series of study in many areas, since materials selection up to projects solutions, as viability, machining techniques, etc. becoming a study target in Physics and Material Engineer.

The first prototype is currently under development and we are working with others important works, with the purpose of obtain a better solution for the problem.

\section{Acknowledgement}

We acknowledge support from CNPQ and FAPESP, projects 2014/23399-9 and 2012/10127-5.

EKIN, J. W. Experimental Techniques for Low-Temperature Measurements Cryostat Design, Material Properties, and Superconductor Critical-Current Testing. Oxford University Press, $1^{\mathrm{a}}$ ed., 2006.

O'HANLON, J. F. A user's guide to vacuum technology. Wiley-Interscience, $3^{\mathrm{a}}$ ed., 2003

ROTH, A. Vacuum technology. North Holland, $1^{\mathrm{a}}$ ed., 1976. 